\title{
SUMS OF FOUR PRIME CUBES IN SHORT INTERVALS
}

\author{
ALESSANDRO LANGUASCO and ALESSANDRO ZACCAGNINI
}

\begin{abstract}
Aвstract. We prove that a suitable asymptotic formula for the average number of representations of integers $n=p_{1}^{3}+p_{2}^{3}+p_{3}^{3}+p_{4}^{3}$, where $p_{1}, p_{2}, p_{3}, p_{4}$ are prime numbers, holds in intervals shorter than the the ones previously known.
\end{abstract}

\section{INTRODUCTION}

Let $N$ be a sufficiently large integer and $1 \leq H \leq N$ an integer. Let

$$
\sum_{n=N+1}^{N+H} r(n), \quad \text { where } \quad r(n)=\sum_{n=p_{1}^{3}+p_{2}^{3}+p_{3}^{3}+p_{4}^{3}} \log p_{1} \log p_{2} \log p_{3} \log p_{4},
$$

be a suitable short interval average of the number of representation of an integer as a sum of four prime cubes. The problem of representing integers as sum of prime cubes is quite an old one; we recall that Hua [4]-[5] stated that almost all positive integers satisfying some necessary congruence conditions are the sum of five prime cubes and that Daveport [2] proved that almost all positive integers are the sum of four positive cubes. More recent results on the positive proportions of integers that are the sum of four prime cubes were obtained by Roth [13], Ren [11] and Liu [9]. In fact, see Brüdern [1], it is conjectured that all sufficiently large integers satisfying some necessary congruence conditions are the sum of four prime cubes. Here we prove that

Theorem 1. Let $N \geq 2,1 \leq H \leq N$ be integers. Then, for every $\varepsilon>0$, there exists $C=C(\varepsilon)>0$ such that

$$
\sum_{n=N+1}^{N+H} r(n)=\Gamma\left(\frac{4}{3}\right)^{3} H N^{1 / 3}+\sigma\left(H N^{1 / 3} \exp \left(-C\left(\frac{\log N}{\log \log N}\right)^{1 / 3}\right)\right) \quad \text { as } N \rightarrow \infty,
$$

uniformly for $N^{13 / 18+\varepsilon} \leq H \leq N^{1-\varepsilon}$, where $\Gamma$ is Euler's function.

This should be compared with a recent result about the positive proportion of such integers in short intervals by Liu-Zhao [8] which holds for $H=N^{17 / 18}$. As an immediate consequence of Theorem 1 we can say that, for $N$ sufficiently large, every interval of size larger than $N^{13 / 18+\varepsilon}$ contains the expected amount of integers which are a sum of four prime cubes. We remark that this level is essentially optimal given the known density estimates for the non trivial zeroes of the Riemann zeta function. Assuming the Riemann Hypothesis (RH) holds we can further improve the size of $H$.

2010 Mathematics Subject Classification. Primary 11 $\mathrm{P}_{32}$; Secondary $11 \mathrm{P}_{55},{ }_{11} \mathrm{P}_{5}$.

Key words and phrases. Waring-Goldbach problem, Hardy-Littlewood method. 
Theorem 2. Let $\varepsilon>0, N \geq 2,1 \leq H \leq N$ be integers and assume the Riemann Hypothesis $(R H)$ holds. Then there exists a constant $B>3 / 2$ such that

$$
\sum_{n=N+1}^{N+H} r(n)=\Gamma\left(\frac{4}{3}\right)^{3} H N^{1 / 3}+\circlearrowleft\left(\frac{H^{2}}{N^{2 / 3}}+H^{3 / 4} N^{5 / 12+\varepsilon}+H^{1 / 2} N^{2 / 3}(\log N)^{B}+N(\log N)^{3}\right)
$$

as $N \rightarrow \infty$, uniformly for $\infty\left(N^{2 / 3} L^{2 B}\right) \leq H \leq o(N)$, where $f=\infty(g)$ means $g=o(f)$ and $\Gamma$ is Euler's function.

As an immediate consequence of Theorem 2 we can say that, for $N$ sufficiently large, every interval of size larger than $N^{2 / 3+\varepsilon}$ contains the expected amount of integers which are a sum of four prime cubes. We remark that this level is essentially optimal given the spacing of the cubic sequence.

In both the proofs of Theorems 1,2 we will use the original Hardy-Littlewood generating functions to exploit the easier main term treatment they allow (comparing with the one which would follow using Lemmas 2.3 and 2.9 of Vaughan [14]).

\section{Setting}

Let $e(\alpha)=e^{2 \pi i \alpha}, \alpha \in[-1 / 2,1 / 2], L=\log N, z=1 / N-2 \pi i \alpha$,

$$
\widetilde{S}_{\ell}(\alpha)=\sum_{n=1}^{\infty} \Lambda(n) e^{-n^{\ell} / N} e\left(n^{\ell} \alpha\right) \quad \text { and } \quad \widetilde{V}_{\ell}(\alpha)=\sum_{p=2}^{\infty} \log p e^{-p^{\ell} / N} e\left(p^{\ell} \alpha\right) .
$$

We remark that

$$
|z|^{-1} \ll \min \left(N,|\alpha|^{-1}\right)
$$

We further set

$$
U(\alpha, H)=\sum_{m=1}^{H} e(m \alpha)
$$

and, moreover, we also have the usual numerically explicit inequality

$$
|U(\alpha, H)| \leq \min \left(H ;|\alpha|^{-1}\right),
$$

see, e.g., on page 39 of Montgomery [10]. We list now the needed preliminary results.

Lemma 1 (Lemma 3 of [6]). Let $\ell \geq 1$ be an integer. Then $\left|\widetilde{S}_{\ell}(\alpha)-\widetilde{V}_{\ell}(\alpha)\right| \ll_{\ell} N^{1 /(2 \ell)}$.

Lemma 2. Let $\ell \geq 1$ be an integer, $N \geq 2$ and $\alpha \in[-1 / 2,1 / 2]$. Then

$$
\widetilde{S}_{\ell}(\alpha)=\frac{\Gamma(1 / \ell)}{\ell z^{1 / \ell}}-\frac{1}{\ell} \sum_{\rho} z^{-\rho / \ell} \Gamma(\rho / \ell)+\sigma_{\ell}(1),
$$

where $\rho=\beta+$ ir runs over the non-trivial zeros of $\zeta(s)$.

Proof. It follows the line of Lemma 2 of [7]; we just correct an oversight in its proof. In eq. (5) on page 48 of [7] the term $-\sum_{m=1}^{\ell \sqrt{3} / 4} \Gamma(-2 m / \ell) z^{2 m / \ell}$ is missing. Its estimate is trivially $\ll_{\ell}|z|^{\sqrt{3} / 2} \ll_{\ell} 1$. Hence such an oversight does not affect the final result of Lemma 2 of [7].

Lemma 3 (Lemma 4 of [7]). Let $N$ be a positive integer and $\mu>0$. Then

$$
\int_{-1 / 2}^{1 / 2} z^{-\mu} e(-n \alpha) \mathrm{d} \alpha=e^{-n / N} \frac{n^{\mu-1}}{\Gamma(\mu)}+\sigma_{\mu}\left(\frac{1}{n}\right)
$$


uniformly for $n \geq 1$.

Lemma 4. Let $\varepsilon$ be an arbitrarily small positive constant, $\ell \geq 1$ be an integer, $N$ be a sufficiently large integer and $L=\log N$. Then there exists a positive constant $c_{1}=c_{1}(\varepsilon)$, which does not depend on $\ell$, such that

$$
\int_{-\xi}^{\xi}\left|\widetilde{S}_{\ell}(\alpha)-\frac{\Gamma(1 / \ell)}{\ell z^{1 / \ell}}\right|^{2} \mathrm{~d} \alpha \ll_{\ell} N^{2 / \ell-1} \exp \left(-c_{1}\left(\frac{L}{\log L}\right)^{1 / 3}\right)
$$

uniformly for $0 \leq \xi<N^{-1+5 /(6 \ell)-\varepsilon}$. Assuming $R H$ we get

$$
\int_{-\xi}^{\xi}\left|\widetilde{S}_{\ell}(\alpha)-\frac{\Gamma(1 / \ell)}{\ell z^{1 / \ell}}\right|^{2} \mathrm{~d} \alpha \ll_{\ell} N^{1 / \ell} \xi L^{2}
$$

uniformly for $0 \leq \xi \leq 1 / 2$.

Proof. It follows the line of Lemma 3 of [7] and Lemma 1 of [6]; we just correct an oversight in their proofs which is based on Lemma 2 above. Both eq. (8) on page 49 of [7] and eq. (6) on page 423 of [6] should read as

$$
\int_{1 / N}^{\xi}\left|\sum_{\rho: \gamma>0} z^{-\rho / \ell} \Gamma(\rho / \ell)\right|^{2} \mathrm{~d} \alpha \leq \sum_{k=1}^{K} \int_{\eta}^{2 \eta}\left|\sum_{\rho: \gamma>0} z^{-\rho / \ell} \Gamma(\rho / \ell)\right|^{2} \mathrm{~d} \alpha
$$

where $\eta=\eta_{k}=\xi / 2^{k}, 1 / N \leq \eta \leq \xi / 2$ and $K$ is a suitable integer satisfying $K=\mathcal{O}(L)$. The remaining part of the proofs are left untouched. Hence such oversights do not affect the final result of Lemma 3 of [7] and Lemma 1 of [6].

In the unconditional case a crucial role is played by

Lemma 5 (Hua). Let $N$ be sufficiently large, $\ell, k$ integers, $\ell \geq 1,1 \leq k \leq \ell$. There exists a suitable positive constant $A=A(\ell, k)$ such that

$$
\int_{-1 / 2}^{1 / 2}\left|\widetilde{S}_{\ell}(\alpha)\right|^{2^{k}} \mathrm{~d} \alpha \ll_{k, \ell} N^{\left(2^{k}-k\right) / \ell} L^{A} \quad \text { and } \quad \int_{-1 / 2}^{1 / 2}\left|\widetilde{V}_{\ell}(\alpha)\right|^{2^{k}} \mathrm{~d} \alpha \ll_{k, \ell} N^{\left(2^{k}-k\right) / \ell} L^{A} .
$$

Proof. We just prove the first part since the second one follows immediately by remarking that the primes are supported on a thinner set than the prime powers. Let $P=(2 N L / \ell)^{1 / \ell}$. A direct estimate gives $\widetilde{S}_{\ell}(\alpha)=\sum_{n \leq P} \Lambda(n) e^{-n^{\ell} / N} e\left(n^{\ell} \alpha\right)+\sigma_{\ell}\left(L^{1 / \ell}\right)$. Recalling that the Prime Number Theorem implies $S_{\ell}(\alpha ; t):=\sum_{n \leq t} \Lambda(n) e\left(n^{\ell} \alpha\right) \ll t$, a partial integration argument gives

$$
\sum_{n \leq P} \Lambda(n) e^{-n^{\ell} / N} e\left(n^{\ell} \alpha\right)=-\frac{\ell}{N} \int_{1}^{P} t^{\ell-1} e^{-t^{\ell} / N} S_{\ell}(\alpha ; t) \mathrm{d} t+\sigma_{\ell}\left(L^{1 / \ell}\right) .
$$

Using the inequality $(|a|+|b|)^{2^{k}} \ll_{k}|a|^{2^{k}}+|b|^{2^{k}}$, Hölder's inequality and interchanging the integrals, we get that

$$
\begin{aligned}
\int_{-1 / 2}^{1 / 2}\left|\widetilde{S}_{\ell}(\alpha)\right|^{2^{k}} \mathrm{~d} \alpha \ll_{k, \ell} \int_{-1 / 2}^{1 / 2}\left|\frac{1}{N} \int_{1}^{P} t^{\ell-1} e^{-t^{\ell} / N} S_{\ell}(\alpha ; t) \mathrm{d} t\right|^{2^{k}} \mathrm{~d} \alpha+L^{2^{k} / \ell} \\
\quad \ll_{k, \ell} \frac{1}{N^{2^{k}}}\left(\int_{1}^{P} t^{\ell-1} e^{-t^{\ell} / N} \mathrm{~d} t\right)^{2^{k}-1}\left(\int_{1}^{P} t^{\ell-1} e^{-t^{\ell} / N} \int_{-1 / 2}^{1 / 2}\left|S_{\ell}(\alpha ; t)\right|^{2^{k}} \mathrm{~d} \alpha \mathrm{d} t\right)+L^{2^{k} / \ell} .
\end{aligned}
$$

Theorem 4 of Hua [5] implies, remarking that the von Mangoldt function is supported on a thinner set than the integers and inserts a logarithmic weight whose total contribution can be inserted in the power of $L$, that there exists a positive constant $B=B(\ell, k)$ such 
that $\int_{-1 / 2}^{1 / 2}\left|S_{\ell}(\alpha ; t)\right|^{2^{k}} \mathrm{~d} \alpha \ll_{k, \ell} t^{2^{k}-k}(\log t)^{B}$. Using such an estimate and remarking that $\int_{1}^{P} t^{\ell-1} e^{-t^{\ell} / N} \mathrm{~d} t \ll_{\ell} N$, we obtain that

$$
\int_{-1 / 2}^{1 / 2}\left|\widetilde{S}_{\ell}(\alpha)\right|^{2^{k}} \mathrm{~d} \alpha \ll_{k, \ell} \frac{1}{N} \int_{1}^{P} t^{\ell-1+2^{k}-k} e^{-t^{\ell} / N}(\log t)^{B} \mathrm{~d} t+L^{2^{k} / \ell} \ll_{k, \ell} N^{\left(2^{k}-k\right) / \ell} L^{B+\left(2^{k}-k\right) / \ell}
$$

by a direct computation. This proves the first part of the lemma.

In fact the argument used in the proof of Lemma 5 can be used to derive other estimates on $\widetilde{S}_{\ell}(\alpha)$ from the ones on $S_{\ell}(\alpha ; t)$. Another instance of this fact is the following lemma about the truncated fourth-mean average of $\widetilde{S}_{\ell}(\alpha)$ which is based on a result by Robert-Sargos [12].

Lemma 6. Let $N \in \mathbb{N}, \varepsilon>0, \ell>1$ and $\tau>0$. Then we have

$$
\int_{-\tau}^{\tau}\left|\widetilde{S}_{\ell}(\alpha)\right|^{4} \mathrm{~d} \alpha \ll\left(\tau N^{2 / \ell}+N^{4 / \ell-1}\right) N^{\varepsilon} \quad \text { and } \quad \int_{-\tau}^{\tau}\left|\widetilde{V}_{\ell}(\alpha)\right|^{4} \mathrm{~d} \alpha \ll\left(\tau N^{2 / \ell}+N^{4 / \ell-1}\right) N^{\varepsilon} .
$$

Proof. We can argue as in the proof of Lemma [5 using Lemma 4 of [3] on $S_{\ell}(\alpha ; t)=$ $\sum_{n \leq t} \Lambda(n) e\left(n^{\ell} \alpha\right)$ instead of Theorem 4 of Hua [5].

The last lemma is a consequence of Lemma 6 .

Lemma 7. Let $N \in \mathbb{N}, \varepsilon>0, \ell>2, c \geq 1$ and $N^{-c} \leq \omega \leq N^{2 / \ell-1}$. Then we have

$$
\left(\int_{-1 / 2}^{-\omega}+\int_{\omega}^{1 / 2}\right)\left|\widetilde{S}_{\ell}(\alpha)\right|^{4} \frac{\mathrm{d} \alpha}{|\alpha|} \ll \frac{N^{4 / \ell-1+\varepsilon}}{\omega} \text { and }\left(\int_{-1 / 2}^{-\omega}+\int_{\omega}^{1 / 2}\right)\left|\widetilde{V}_{\ell}(\alpha)\right|^{4} \frac{\mathrm{d} \alpha}{|\alpha|} \ll \frac{N^{4 / \ell-1+\varepsilon}}{\omega} \text {. }
$$

Proof. By partial integration and Lemma 6 we get that

$$
\begin{aligned}
\int_{\omega}^{1 / 2}\left|\widetilde{S}_{\ell}(\alpha)\right|^{4} \frac{\mathrm{d} \alpha}{\alpha} & \ll \frac{1}{\omega} \int_{-\omega}^{\omega}\left|\widetilde{S}_{\ell}(\alpha)\right|^{4} \mathrm{~d} \alpha+\int_{-1 / 2}^{1 / 2}\left|\widetilde{S}_{\ell}(\alpha)\right|^{4} \mathrm{~d} \alpha+\int_{\omega}^{1 / 2}\left(\int_{-\xi}^{\xi}\left|\widetilde{S}_{\ell}(\alpha)\right|^{4} \mathrm{~d} \alpha\right) \frac{\mathrm{d} \xi}{\xi^{2}} \\
& \ll \frac{1}{\omega}\left(\omega N^{2 / \ell}+N^{4 / \ell-1}\right) N^{\varepsilon}+N^{2 / \ell+\varepsilon}+N^{\varepsilon} \int_{\omega}^{1 / 2} \frac{\xi N^{2 / \ell}+N^{4 / \ell-1}}{\xi^{2}} \mathrm{~d} \xi \\
& \ll N^{2 / \ell+\varepsilon}|\log (2 \omega)|+\frac{N^{4 / \ell-1+\varepsilon}}{\omega} \ll \frac{N^{4 / \ell-1+\varepsilon}}{\omega}
\end{aligned}
$$

since $N^{-c} \leq \omega \leq N^{2 / \ell-1}$. A similar computation proves the result in $[-1 / 2,-\omega]$ too. The estimate on $\widetilde{V}_{\ell}(\alpha)$ can be obtained analogously.

\section{The UnCONDitional CASE}

Let $H>2 B$, where

$$
B=N^{2 \varepsilon} .
$$

Letting $I(B, H):=[-1 / 2,-B / H] \cup[B / H, 1 / 2]$, and recalling (1), we have

$$
\begin{aligned}
\sum_{n=N+1}^{N+H} e^{-n / N} r(n)= & \int_{-1 / 2}^{1 / 2} \widetilde{V}_{3}(\alpha)^{4} U(-\alpha, H) e(-N \alpha) \mathrm{d} \alpha \\
= & \int_{-B / H}^{B / H} \widetilde{S}_{3}(\alpha)^{4} U(-\alpha, H) e(-N \alpha) \mathrm{d} \alpha+\int_{I(B, H)} \widetilde{S}_{3}(\alpha)^{4} U(-\alpha, H) e(-N \alpha) \mathrm{d} \alpha \\
& +\int_{-1 / 2}^{1 / 2}\left(\widetilde{V}_{3}(\alpha)^{4}-\widetilde{S}_{3}(\alpha)^{4}\right) U(-\alpha, H) e(-N \alpha) \mathrm{d} \alpha=I_{1}+I_{2}+I_{3},
\end{aligned}
$$


say. Now we evaluate these terms.

3.1. Estimation of $I_{2}$. Using (3) and Lemma 7 with $\omega=B / H$ and $\ell=3$, we obtain

$$
I_{2} \ll \int_{B / H}^{1 / 2}\left|\widetilde{S}_{3}(\alpha)\right|^{4} \frac{\mathrm{d} \alpha}{\alpha} \ll \frac{H}{B} N^{1 / 3+\varepsilon}
$$

provided that $H \gg N^{1 / 3} B$.

3.2. Estimation of $I_{3}$. Clearly

$$
\begin{aligned}
\left|\widetilde{V}_{3}(\alpha)^{4}-\widetilde{S}_{3}(\alpha)^{4}\right| & =\left|\widetilde{V}_{3}(\alpha)-\widetilde{S}_{3}(\alpha)\right|\left|\widetilde{V}_{3}(\alpha)^{3}+\widetilde{V}_{3}(\alpha)^{2} \widetilde{S}_{3}(\alpha)+\widetilde{V}_{3}(\alpha) \widetilde{S}_{3}(\alpha)^{2}+\widetilde{S}_{3}(\alpha)^{3}\right| \\
& \ll\left|\widetilde{V}_{3}(\alpha)-\widetilde{S}_{3}(\alpha)\right|\left(\left|\widetilde{V}_{3}(\alpha)\right|+\left|\widetilde{S}_{3}(\alpha)\right|\right)^{3} \\
& \ll\left|\widetilde{V}_{3}(\alpha)-\widetilde{S}_{3}(\alpha)\right| \max \left(\left|\widetilde{V}_{3}(\alpha)\right|^{3} ;\left|\widetilde{S}_{3}(\alpha)\right|^{3}\right) .
\end{aligned}
$$

Hence by Lemma 1 we have

$$
I_{3} \ll N^{1 / 6} \int_{-1 / 2}^{1 / 2}\left(\left|\widetilde{V}_{3}(\alpha)\right|^{3}+\left|\widetilde{S}_{3}(\alpha)\right|^{3}\right)|U(-\alpha, H)| \mathrm{d} \alpha=N^{1 / 6}\left(K_{1}+K_{2}\right),
$$

say. By (3) we get

$$
K_{2} \ll H \int_{-1 / H}^{1 / H}\left|\widetilde{S}_{3}(\alpha)\right|^{3} \mathrm{~d} \alpha+\left(\int_{-1 / 2}^{-1 / H}+\int_{1 / H}^{1 / 2}\right)\left|\widetilde{S}_{3}(\alpha)\right|^{3} \frac{\mathrm{d} \alpha}{|\alpha|}=K_{2,1}+K_{2,2},
$$

say. Using the Hölder inequality and Lemma 6 with $\tau=1 / H$ and $\ell=3$ we get

$$
K_{2,1} \ll H^{3 / 4}\left(\int_{-1 / H}^{1 / H}\left|\widetilde{S}_{3}(\alpha)\right|^{4} \mathrm{~d} \alpha\right)^{3 / 4} \ll H^{3 / 4} N^{1 / 4+\varepsilon},
$$

provided that $H \gg N^{1 / 3}$. Using the Hölder inequality and Lemma 7 with $\omega=1 / H$ and $\ell=3$ we get

$$
K_{2,2} \ll\left(\int_{1 / H}^{1 / 2}\left|\widetilde{S}_{3}(\alpha)\right|^{4} \frac{\mathrm{d} \alpha}{\alpha}\right)^{3 / 4}\left(\int_{1 / H}^{1 / 2} \frac{\mathrm{d} \alpha}{\alpha}\right)^{1 / 4} \ll\left(H N^{1 / 3+\varepsilon}\right)^{3 / 4} L^{1 / 4} \ll H^{3 / 4} N^{1 / 4+\varepsilon},
$$

provided that $H \gg N^{1 / 3}$. Combining (8)-(10) we obtain

$$
K_{2} \ll H^{3 / 4} N^{1 / 4+\varepsilon},
$$

provided that $H \gg N^{1 / 3}$. An analogous computation gives

$$
K_{1} \ll H^{3 / 4} N^{1 / 4+\varepsilon},
$$

and, by (17) and (11)-(12), we can finally write

$$
I_{3} \ll H^{3 / 4} N^{5 / 12+\varepsilon},
$$

provided that $H \gg N^{1 / 3}$. 
3.3. Evaluation of $I_{1}$. Since $\Gamma(4 / 3)=(1 / 3) \Gamma(1 / 3)$, we have that

$$
\begin{aligned}
I_{1} & =\int_{-B / H}^{B / H} \frac{\Gamma(4 / 3)^{4}}{z^{4 / 3}} U(-\alpha, H) e(-N \alpha) \mathrm{d} \alpha+\int_{-B / H}^{B / H}\left(\widetilde{S}_{3}(\alpha)^{4}-\frac{\Gamma(4 / 3)^{4}}{z^{4 / 3}}\right) U(-\alpha, H) e(-N \alpha) \mathrm{d} \alpha \\
& =J_{1}+J_{2},
\end{aligned}
$$

say. By (2)-( (3) and Lemma 3, a direct calculation gives

$$
\begin{aligned}
J_{1} & =\Gamma\left(\frac{4}{3}\right)^{3} \sum_{n=N+1}^{N+H} e^{-n / N} n^{1 / 3}+\odot\left(\frac{H}{N}\right)+\odot\left(\int_{B / H}^{1 / 2} \frac{\mathrm{d} \alpha}{\alpha^{7 / 3}}\right) \\
& =\frac{\Gamma(4 / 3)^{3}}{e} \sum_{n=N+1}^{N+H} n^{1 / 3}+\odot\left(\frac{H}{N}+\frac{H^{2}}{N^{2 / 3}}+\frac{H^{4 / 3}}{B^{4 / 3}}\right) \\
& =\Gamma\left(\frac{4}{3}\right)^{3} \frac{H N^{1 / 3}}{e}+\odot\left(\frac{H^{4 / 3}}{B^{4 / 3}}+N^{1 / 3}\right) .
\end{aligned}
$$

From now on, we denote $\widetilde{E}_{3}(\alpha):=\widetilde{S}_{3}(\alpha)-\frac{\Gamma(4 / 3)}{z^{1 / 3}}$. By $f^{2}-g^{2}=2 g(f-g)+(f-g)^{2}$, (2) and $\widetilde{S}_{3}(\alpha) \ll N^{1 / 3}$ we get

$$
\begin{aligned}
\widetilde{S}_{3}(\alpha)^{4}-\frac{\Gamma(4 / 3)^{4}}{z^{4 / 3}} & =\left(\widetilde{S}_{3}(\alpha)^{2}+\frac{\Gamma(4 / 3)^{2}}{z^{2 / 3}}\right)\left(\widetilde{S}_{3}(\alpha)^{2}-\frac{\Gamma(4 / 3)^{2}}{z^{2 / 3}}\right) \\
& =\left(\widetilde{S}_{3}(\alpha)^{2}+\frac{\Gamma(4 / 3)^{2}}{z^{2 / 3}}\right)\left(2 \frac{\Gamma(4 / 3)}{z^{1 / 3}} \widetilde{E}_{3}(\alpha)+\widetilde{E}_{3}(\alpha)^{2}\right) \\
& \ll\left|\widetilde{S}_{3}(\alpha)\right|^{2} \frac{\left|\widetilde{E}_{3}(\alpha)\right|}{|z|^{1 / 3}}+\frac{\left|\widetilde{E}_{3}(\alpha)\right|}{|z|}+N^{2 / 3}\left|\widetilde{E}_{3}(\alpha)\right|^{2} .
\end{aligned}
$$

Using (16) and (21) we get

$$
\begin{aligned}
J_{2} & \ll H \int_{-B / H}^{B / H}\left|\widetilde{S}_{3}(\alpha)\right|^{2} \frac{\left|\widetilde{E}_{3}(\alpha)\right|}{|z|^{1 / 3}} \mathrm{~d} \alpha+H \int_{-B / H}^{B / H} \frac{\left|\widetilde{E}_{3}(\alpha)\right|}{|z|} \mathrm{d} \alpha+H N^{2 / 3} \int_{-B / H}^{B / H}\left|\widetilde{E}_{3}(\alpha)\right|^{2} \mathrm{~d} \alpha \\
& =H\left(E_{1}+E_{2}+N^{2 / 3} E_{3}\right),
\end{aligned}
$$

say. By (3) and Lemma 4 we obtain that, for every $\varepsilon>0$, there exists $c_{1}=c_{1}(\varepsilon)>0$ such that

$$
E_{3} \ll N^{-1 / 3} \exp \left(-c_{1}\left(\frac{L}{\log L}\right)^{1 / 3}\right)
$$

provided that $B / H \leq N^{-13 / 18-\varepsilon}$, i.e., $H \geq B N^{13 / 18+\varepsilon}$. By the Cauchy-Schwarz inequality, (2) and (18) we obtain that, for every $\varepsilon>0$, there exists $c_{1}=c_{1}(\varepsilon)>0$ such that

$$
E_{2} \ll E_{3}^{1 / 2}\left(\int_{-B / H}^{B / H} \frac{\mathrm{d} \alpha}{|z|^{2}}\right)^{1 / 2} \ll E_{3}^{1 / 2} N^{1 / 2} \ll N^{1 / 3} \exp \left(-\frac{c_{1}}{2}\left(\frac{L}{\log L}\right)^{1 / 3}\right),
$$

provided that $H \geq B N^{13 / 18+\varepsilon}$. By using twice the Cauchy-Schwarz inequality, Lemma 5, (2) and (18) we obtain that, for every $\varepsilon>0$, there exists $c_{1}=c_{1}(\varepsilon)>0$ such that

$$
\begin{aligned}
E_{1} & \ll E_{3}^{1 / 2}\left(\int_{-B / H}^{B / H} \frac{\left|\widetilde{S}_{3}(\alpha)\right|^{4}}{|z|^{2 / 3}} \mathrm{~d} \alpha\right)^{1 / 2} \ll E_{3}^{1 / 2}\left(\int_{-1 / 2}^{1 / 2}\left|\widetilde{S}_{3}(\alpha)\right|^{8} \mathrm{~d} \alpha\right)^{1 / 4}\left(\int_{-B / H}^{B / H} \frac{\mathrm{d} \alpha}{|z|^{4 / 3}}\right)^{1 / 4} \\
& \ll E_{3}^{1 / 2} N^{1 / 2} L^{A / 4} \ll N^{1 / 3} \exp \left(-\frac{c_{1}}{4}\left(\frac{L}{\log L}\right)^{1 / 3}\right),
\end{aligned}
$$


provided that $H \geq B N^{13 / 18+\varepsilon}$. Hence by $(\sqrt[17)]{17}(\sqrt{20})$ we finally can write that, for every $\varepsilon>0$, there exists $c_{1}=c_{1}(\varepsilon)>0$ such that

$$
J_{2} \ll H N^{1 / 3} \exp \left(-\frac{c_{1}}{4}\left(\frac{L}{\log L}\right)^{1 / 3}\right),
$$

provided that $H \geq B N^{13 / 18+\varepsilon}$. Summing up, by (14)-(15) and (21) we have that, for every $\varepsilon>0$, there exists $c_{1}=c_{1}(\varepsilon)>0$ such that

$$
I_{1}=\Gamma\left(\frac{4}{3}\right)^{3} \frac{H N^{1 / 3}}{e}+\odot\left(H N^{1 / 3} \exp \left(-\frac{c_{1}}{4}\left(\frac{L}{\log L}\right)^{1 / 3}\right)\right)
$$

provided that $H \geq B N^{13 / 18+\varepsilon}$.

3.4. Final words. Summing up, by (5)-(6), (13) and (22) we have that, for every $\varepsilon>0$, there exists $c_{1}=c_{1}(\varepsilon)>0$ such that

$$
\sum_{n=N+1}^{N+H} e^{-n / N} r(n)=\Gamma\left(\frac{4}{3}\right)^{3} \frac{H N^{1 / 3}}{e}+\circlearrowleft\left(H N^{1 / 3} \exp \left(-\frac{c_{1}}{4}\left(\frac{L}{\log L}\right)^{1 / 3}\right)+\frac{H}{B} N^{1 / 3+\varepsilon}\right)
$$

provided that $H \geq B N^{13 / 18+\varepsilon}$. The second error term is dominated by the first one since $B=N^{2 \varepsilon}$ by (4). Hence we can write that, for every $\varepsilon>0$, there exists $C=C(\varepsilon)>0$ such that

$$
\sum_{n=N+1}^{N+H} e^{-n / N} r(n)=\Gamma\left(\frac{4}{3}\right)^{3} \frac{H N^{1 / 3}}{e}+\odot\left(H N^{1 / 3} \exp \left(-C\left(\frac{L}{\log L}\right)^{1 / 3}\right)\right)
$$

provided that $H \geq N^{13 / 18+3 \varepsilon}$. From $e^{-n / N}=e^{-1}+\mathcal{O}(H / N)$ for $n \in[N+1, N+H], 1 \leq H \leq N$, we get that, for every $\varepsilon>0$, there exists $C=C(\varepsilon)>0$ such that

$$
\sum_{n=N+1}^{N+H} r(n)=\Gamma\left(\frac{4}{3}\right)^{3} H N^{1 / 3}+\odot\left(H N^{1 / 3} \exp \left(-C\left(\frac{L}{\log L}\right)^{1 / 3}\right)\right)+\odot\left(\frac{H}{N} \sum_{n=N+1}^{N+H} r(n)\right)
$$

provided that $H \geq N^{13 / 18+3 \varepsilon}$ and $H \leq N$. Using $e^{n / N} \leq e^{2}$ and (23), the last error term is $\ll H^{2} N^{-2 / 3}$. Hence we get that, for every $\varepsilon>0$, there exists $C=C(\varepsilon)>0$ such that

$$
\sum_{n=N+1}^{N+H} r(n)=\Gamma\left(\frac{4}{3}\right)^{3} H N^{1 / 3}+O\left(H N^{1 / 3} \exp \left(-C\left(\frac{L}{\log L}\right)^{1 / 3}\right)\right),
$$

provided that $N^{13 / 18+3 \varepsilon} \leq H \leq N^{1-\varepsilon}$. Theorem 1 follows by rescaling $\varepsilon$.

\section{The conditional case}

From now on we assume the Riemann Hypothesis holds. Comparing with section 3 we can simplify the setting. Recalling (11) and $\Gamma(4 / 3)=(1 / 3) \Gamma(1 / 3)$, we have

$$
\begin{aligned}
\sum_{n=N+1}^{N+H} e^{-n / N} r(n)= & \int_{-1 / 2}^{1 / 2} \widetilde{V}_{3}(\alpha)^{4} U(-\alpha, H) e(-N \alpha) \mathrm{d} \alpha \\
= & \Gamma\left(\frac{4}{3}\right)^{4} \int_{-1 / 2}^{1 / 2} \frac{U(-\alpha, H)}{z^{4 / 3}} e(-N \alpha) \mathrm{d} \alpha \\
& +\int_{-1 / 2}^{1 / 2}\left(\widetilde{S}_{3}(\alpha)^{4}-\frac{\Gamma(4 / 3)^{4}}{z^{4 / 3}}\right) U(-\alpha, H) e(-N \alpha) \mathrm{d} \alpha
\end{aligned}
$$




$$
\begin{aligned}
& +\int_{-1 / 2}^{1 / 2}\left(\widetilde{V}_{3}(\alpha)^{4}-\widetilde{S}_{3}(\alpha)^{4}\right) U(-\alpha, H) e(-N \alpha) \mathrm{d} \alpha \\
=\mathscr{F}_{1}+\mathscr{J}_{2}+\mathscr{F}_{3}, &
\end{aligned}
$$

say. Now we evaluate these terms.

4.1. Evaluation of $\mathscr{F}_{1}$. By Lemma 3 , a direct calculation gives

$$
\begin{aligned}
\mathscr{g}_{1} & =\Gamma\left(\frac{4}{3}\right)^{3} \sum_{n=N+1}^{N+H} e^{-n / N} n^{1 / 3}+\circlearrowleft\left(\frac{H}{N}\right)=\frac{\Gamma(4 / 3)^{3}}{e} \sum_{n=N+1}^{N+H} n^{1 / 3}+\circlearrowleft\left(\frac{H}{N}+\frac{H^{2}}{N^{2 / 3}}\right) \\
& =\Gamma\left(\frac{4}{3}\right)^{3} \frac{H N^{1 / 3}}{e}+\odot\left(\frac{H^{2}}{N^{2 / 3}}+N^{1 / 3}\right) .
\end{aligned}
$$

4.2. Estimate of $\mathscr{F}_{2}$. Recall that $\widetilde{E}_{3}(\alpha):=\widetilde{S}_{3}(\alpha)-\frac{\Gamma(4 / 3)}{z^{1 / 3}}$. Using $f^{2}-g^{2}=2 g(f-g)+(f-g)^{2}$ we can write that

$$
\begin{aligned}
\widetilde{S}_{3}(\alpha)^{4}-\frac{\Gamma(4 / 3)^{4}}{z^{4 / 3}} & =\left(\widetilde{S}_{3}(\alpha)^{2}+\frac{\Gamma(4 / 3)^{2}}{z^{2 / 3}}\right)\left(\widetilde{S}_{3}(\alpha)^{2}-\frac{\Gamma(4 / 3)^{2}}{z^{2 / 3}}\right) \\
& \ll\left(\left|\widetilde{S}_{3}(\alpha)\right|^{2}+|z|^{-2 / 3}\right)\left(|z|^{-1 / 3}\left|\widetilde{E}_{3}(\alpha)\right|+\left|\widetilde{E}_{3}(\alpha)\right|^{2}\right)
\end{aligned}
$$

Hence

$$
\begin{aligned}
\mathscr{I}_{2} & \ll \int_{-1 / 2}^{1 / 2} \frac{\left|\widetilde{S}_{3}(\alpha)\right|^{2}}{|z|^{1 / 3}}\left|\widetilde{E}_{3}(\alpha)\right||U(-\alpha, H)| \mathrm{d} \alpha+\int_{-1 / 2}^{1 / 2} \frac{\left|\widetilde{E}_{3}(\alpha)\right|}{|z|}|U(-\alpha, H)| \mathrm{d} \alpha \\
& \quad+\int_{-1 / 2}^{1 / 2}\left(\left|\widetilde{S}_{3}(\alpha)\right|^{2}+|z|^{-2 / 3}\right)\left|\widetilde{E}_{3}(\alpha)\right|^{2}|U(-\alpha, H)| \mathrm{d} \alpha \\
= & \mathscr{I}_{1}+\mathscr{I}_{2}+\mathscr{J}_{3},
\end{aligned}
$$

say. Let

$$
\mathscr{E}:=\int_{-1 / 2}^{1 / 2}\left|\widetilde{E}_{3}(\alpha)\right|^{2}|U(-\alpha, H)| \mathrm{d} \alpha .
$$

By (3), Lemma 4 and a partial integration we obtain

$$
\begin{aligned}
\mathscr{E} & \ll H \int_{-1 / H}^{1 / H}\left|\widetilde{E}_{3}(\alpha)\right|^{2} \mathrm{~d} \alpha+\int_{1 / H}^{1 / 2} \frac{\left|\widetilde{E}_{3}(\alpha)\right|^{2}}{\alpha} \mathrm{d} \alpha \\
& \ll H \frac{N^{1 / 3} L^{2}}{H}+N^{1 / 3} L^{2}+\int_{1 / H}^{1 / 2} \frac{N^{1 / 3} L^{2}}{\xi} \mathrm{d} \xi \ll N^{1 / 3} L^{3} .
\end{aligned}
$$

By (21), ${\widetilde{S_{3}}}_{3}(\alpha) \ll N^{1 / 3}$ and (27) we obtain

$$
\mathscr{I}_{3} \ll N^{2 / 3} \mathscr{C} \ll N L^{3}
$$

By the Cauchy-Schwarz inequality, (2)-(3) and (27), we obtain

$$
\begin{aligned}
\mathscr{I}_{2} & \ll \mathscr{E}^{1 / 2}\left(\int_{-1 / 2}^{1 / 2} \frac{|U(-\alpha, H)|}{|z|^{2}} \mathrm{~d} \alpha\right)^{1 / 2} \ll \mathscr{E}^{1 / 2}\left(H N^{2} \int_{-1 / N}^{1 / N} \mathrm{~d} \alpha+H \int_{1 / N}^{1 / H} \frac{\mathrm{d} \alpha}{\alpha^{2}}+\int_{1 / H}^{1 / 2} \frac{\mathrm{d} \alpha}{\alpha^{3}}\right)^{1 / 2} \\
& \ll H^{1 / 2} N^{2 / 3} L^{3 / 2} .
\end{aligned}
$$

By the Cauchy-Schwarz inequality we obtain

$$
\mathscr{I}_{1} \ll \mathscr{E}^{1 / 2}\left(\int_{-1 / 2}^{1 / 2}\left|\widetilde{S}_{3}(\alpha)\right|^{4} \frac{|U(-\alpha, H)|}{|z|^{2 / 3}} \mathrm{~d} \alpha\right)^{1 / 2} .
$$


Again by the Cauchy-Schwarz inequality, (22)-(3) and (27), we obtain

$$
\begin{aligned}
\mathscr{I}_{1} & \ll \mathscr{E}^{1 / 2}\left(\int_{-1 / 2}^{1 / 2}\left|\widetilde{S}_{3}(\alpha)\right|^{8} \mathrm{~d} \alpha\right)^{1 / 4}\left(\int_{-1 / 2}^{1 / 2} \frac{|U(-\alpha, H)|^{2}}{|z|^{4 / 3}} \mathrm{~d} \alpha\right)^{1 / 4} \\
& \ll N^{1 / 6} L^{3 / 2} N^{5 / 12} L^{A / 4}\left(H^{2} N^{4 / 3} \int_{-1 / N}^{1 / N} \mathrm{~d} \alpha+H^{2} \int_{1 / N}^{1 / H} \frac{\mathrm{d} \alpha}{\alpha^{4 / 3}}+\int_{1 / H}^{1 / 2} \frac{\mathrm{d} \alpha}{\alpha^{10 / 3}}\right)^{1 / 4} \\
& \ll H^{1 / 2} N^{2 / 3} L^{3 / 2+A / 4} .
\end{aligned}
$$

Summing up by (26) and (28)-(30), we can finally write that

$$
\mathscr{F}_{2} \ll H^{1 / 2} N^{2 / 3} L^{3 / 2+A / 4}+N L^{3} .
$$

4.3. Estimate of $\mathscr{F}_{3}$. It is clear that $\mathscr{F}_{3}=I_{3}$ of section 3.2 . Hence by $(\sqrt{13})$ we obtain

$$
\mathscr{F}_{3} \ll H^{3 / 4} N^{5 / 12+\varepsilon} \text {. }
$$

4.4. Final words. Summing up, by (24)-(25), (31) and ([32), there exists $B=B(A)>0$ such that we have

$$
\sum_{n=N+1}^{N+H} e^{-n / N} r(n)=\Gamma\left(\frac{4}{3}\right)^{3} \frac{H N^{1 / 3}}{e}+\circlearrowleft\left(\frac{H^{2}}{N^{2 / 3}}+H^{3 / 4} N^{5 / 12+\varepsilon}+H^{1 / 2} N^{2 / 3} L^{B}+N L^{3}\right)
$$

which is an asymptotic formula $\infty\left(N^{2 / 3} L^{2 B}\right) \leq H \leq o(N)$. From $e^{-n / N}=e^{-1}+\circlearrowleft(H / N)$ for $n \in[N+1, N+H], 1 \leq H \leq N$, we get

$$
\sum_{n=N+1}^{N+H} r(n)=\Gamma\left(\frac{4}{3}\right)^{3} H N^{1 / 3}+\circlearrowleft\left(\frac{H^{2}}{N^{2 / 3}}+H^{3 / 4} N^{5 / 12+\varepsilon}+H^{1 / 2} N^{2 / 3} L^{B}+N L^{3}\right)+\circlearrowleft\left(\frac{H}{N} \sum_{n=N+1}^{N+H} r(n)\right) .
$$

Using $e^{n / N} \leq e^{2}$ and ( $(\overline{33})$, the last error term is $\ll H^{2} N^{-2 / 3}+H^{7 / 4} N^{-7 / 12+\varepsilon}+H^{3 / 2} N^{-2 / 3} L^{B}+$ $H L^{3}$. Hence we get

$$
\sum_{n=N+1}^{N+H} r(n)=\Gamma\left(\frac{4}{3}\right)^{3} H N^{1 / 3}+O\left(\frac{H^{2}}{N^{2 / 3}}+H^{3 / 4} N^{5 / 12+\varepsilon}+H^{1 / 2} N^{2 / 3} L^{B}+N L^{3}\right),
$$

uniformly for $\infty\left(N^{2 / 3} L^{2 B}\right) \leq H \leq o(N), B>3 / 2$. Theorem 2 follows.

\section{REFERENCES}

[1] J. Brüdern, A sieve approach to the Waring-Goldbach problem. I. Sums of four cubes, Ann. Sci. École Norm. Sup. (4) 28 (1995), 461-476.

[2] H. Davenport, On Waring's problem for cubes, Acta Math. 71 (1939), 123-143.

[3] A. Gambini, A. Languasco, and A. Zaccagnini, A diophantine approximation problem with two primes and one k-power of a prime, J. Number Theory 188 (2018), 210-228.

[4] L. K. Hua, Some results in the additive prime number theory, Quart. J. Math. Oxford 9 (1938), 68-80.

[5] L. K. Hua, Additive theory of prime numbers, Trans. Math. Monographs, vol. 13, A.M.S., 1965.

[6] A. Languasco and A. Zaccagnini, Short intervals asymptotic formulae for binary problems with primes and powers, II: density 1, Monatsh. Math. 181 (2016), 419-435.

[7] A. Languasco and A. Zaccagnini, Sum of one prime and two squares of primes in short intervals, J. Number Theory 159 (2016), 1945-1960. 
[8] H. Liu and F. Zhao, Density of integers that are the sum of four cubes of primes in short intervals, Acta Math. Hungar. 151 (2017), 8-23.

[9] Z. Liu, Density of the sums of four cubes of primes, J. Number Theory 132 (2012), $735-747$.

[10] H. L. Montgomery, Ten Lectures on the Interface Between Analytic Number Theory and Harmonic Analysis, CBMS Regional Conference Series in Mathematics, vol. 84, A.M.S., 1994.

[11] X. Ren, Sums of four cubes of primes, J. Number Theory 98 (2003), 156-171.

[12] O. Robert and P. Sargos, Three-dimensional exponential sums with monomials, J. reine angew. Math. 591 (2006), 1-20.

[13] K. F. Roth, On Waring's problem for cubes, Proc. London Math. Soc. (2) 53 (1951), 268-279.

[14] R. C. Vaughan, The Hardy-Littlewood method, second ed., Cambridge U. P., 1997.

Alessandro Languasco

Università di Padova

Dipartimento di Matematica

"Tullio Levi-Civita"

Via Trieste 63

35121 Padova, Italy

e-mail: alessandro.languasco@unipd.it
Alessandro Zaccagnini

Università di Parma

Dipartimento di Scienze Matematiche,

Fisiche e Informatiche

Parco Area delle Scienze, 53/a

43124 Parma, Italy

e-mail: alessandro.zaccagnini@unipr.it 